\title{
A COUNTEREXAMPLE TO THE RIGIDITY CONJECTURE FOR RINGS
}

\author{
RAYMOND C. HEITMANN
}

\begin{abstract}
An example is constructed of a local ring and a module of finite type and finite projective dimension over that ring such that the module is not rigid. This shows that the rigidity conjecture is false.
\end{abstract}

\section{INTRODUCTION}

For a ring $R$, an $R$-module $M$ is said to be rigid if for every $R$-module $N$ of finite type, whenever $\operatorname{Tor}_{i}^{R}(M, N)=0$, then $\operatorname{Tor}_{j}^{R}(M, N)=0$ for all $j \geq i$. The concept was developed in 1961 by Auslander, who showed that all modules over unramified regular local rings were rigid [1] and who also demonstrated a number of other results for modules over unramified regular local rings, all of which follow directly from the rigidity property and so would hold more generally if a larger class of modules were known to be rigid. The most notable of these came to be known as the zero divisor conjecture: Let $R$ be a local ring, and let $M$ be an $R$-module of finite type and finite projective dimension. If $x \in R$ is not a zero divisor on $M$, then $x$ is not a zero divisor on $R$. (Auslander's original result used regular sequences in place of nonzero divisors, but this more elementary formulation, which appears in $[4, \mathrm{p} .8]$, is equivalent.)

Auslander also noted the importance of finite projective dimension, giving an example of a nonrigid module of infinite projective dimension; thus, it was natural to focus on regular local rings, which have the property that all modules have finite projective dimension. Auslander's original question was answered in 1966 when Lichtenbaum proved the rigidity conjecture for all regular local rings [5]. However, because of the numerous consequences of rigidity, it seemed natural to hope that the property held for modules of finite projective dimension. Thus, Peskine and Szpiro gave the conjecture its present form in [6]: If $R$ is a Noetherian ring and $M$ is an $R$-module of finite type and finite projective dimension, then $M$ is rigid. In that article they offered conjectures generalizing many of Auslander's results plus many other related conjectures. The conjectures are primarily local in nature, as, for example, a module is rigid if and only if it is locally rigid. For equicharacteristic local rings of characteristic $p$ (both the ring and its residue field have characteristic $p$ ), Peskine and Szpiro were successful in proving a number of the conjectures, including the zero divisor conjecture. Hochster extended this work to equicharacteristic zero, and his CBMS monograph [4] gives a thorough presentation of the entire subject. There was, however, no real progress on rigidity.

More recently, P. Roberts has been able to demonstrate some of the conjectures which had previously eluded proof in the case of local rings of mixed

Received by the editors September 25, 1992.

1991 Mathematics Subject Classification. Primary 13C99, 18G15; Secondary 13D25, 13 H99. 
characteristic [8]. In particular, he has shown that Auslander's zero divisor conjecture and several other consequences of rigidity are valid for all local rings. Thus, while the rigidity conjecture has turned out to be false, many of its implications are true.

It is interesting to note that the example given here is not pathological. It is an affine domain over a field constructed in a generic fashion. It is not a complete intersection, and the conjecture thus is not resolved for those rings. Hochster has suggested to me privately that he does not believe this additional hypothesis will be helpful.

An alternate approach is to view rigidity as a property of the pair $M, N$ rather than just $M$. For regular local rings, necessarily both $M$ and $N$ have finite projective dimension, and so, in this context, it seems appropriate to assume that both modules have finite projective dimension. This question likewise remains open.

\section{THE EXAMPLE}

To settle this conjecture in the negative, we will construct a Noetherian ring $R$ together with $R$-modules $M$ and $N$ such that $p d_{R} M=2, N$ is a module of finite length, $\operatorname{Tor}_{1}^{R}(M, N)=0$, and $\operatorname{Tor}_{2}^{R}(M, N) \neq 0$. Lemma 1 is not really new, and to avoid disturbing the flow of the construction, we will defer its proof to the end of the article.

Lemma 1. There exists an affine $K$-algebra $R$ where $K$ is a field, matrices $X=\left[x_{i j}\right]$ and $Y=\left[y_{i j}\right]$ of dimensions $2 \times 4$ and $4 \times 8$ respectively, and $a$ maximal ideal $P$ of $R$ such that:

(1) $0 \rightarrow R^{2} \stackrel{X}{\longrightarrow} R^{4} \stackrel{Y}{\longrightarrow} R^{8}$ is exact;

(2) $\left\{\bar{x}_{i j}, \bar{y}_{i j}\right\}$ is a linearly independent subset of $P / P^{2}$; and

(3) $R / P=K$

Definition. Let $S=K[s, t] /\left(s^{2}, s t, t^{2}\right)$. Also let $N=S^{2} /((t, 0),(0, s)$, $(s, t)) S$, a length $3 S$-module. Note that $(s, t) N$ is a length 1 submodule.

Lemma 2. There is a homomorphism $R \rightarrow S$ which takes

$$
X \text { to } \bar{X}=\left(\begin{array}{llll}
s & 0 & t & 0 \\
0 & s & 0 & t
\end{array}\right) \text { and } Y \text { to } \bar{Y}=\left(\begin{array}{llllllll}
s & 0 & 0 & 0 & t & 0 & 0 & 0 \\
0 & s & 0 & 0 & 0 & t & 0 & 0 \\
0 & 0 & s & 0 & 0 & 0 & t & 0 \\
0 & 0 & 0 & s & 0 & 0 & 0 & t
\end{array}\right) \text {. }
$$

This map gives $N$ an $R$-module structure.

Proof. It suffices to find a homomorphism $R / P^{2} \rightarrow S$. So we may assume $R=K \oplus P$ where $P$ is a $K$-vector space and $S=K \oplus(s, t) K$. Then a $K$-algebra homomorphism is determined by a linear transformation $P \rightarrow(s, t) K$. Now, as $\left\{\bar{x}_{i j}, \bar{y}_{i j}\right\}$ is linearly independent, there is clearly such a transformation with the desired $\bar{X}, \bar{Y}$.

Theorem 3. With the above notation and $M=R^{8} /\left(R^{4}\right) Y, p d_{R} M=2$ and $\operatorname{Tor}_{1}^{R}(M, N)=0$, but $\operatorname{Tor}_{2}^{R}(M, N) \neq 0$.

Proof. Since $0 \rightarrow R^{2} \stackrel{X}{\longrightarrow} R^{4} \stackrel{Y}{\longrightarrow} R^{8} \rightarrow M$ is a projective resolution of $M$, it 
follows that $p d_{R} M \leq 2$. As each entry of $X$ is in $P$, the injection does not split, and so we actually have equality.

To compute Tor, note that $-\otimes_{R} N=\left(-\otimes_{R} S\right) \otimes_{S} N$. Thus we can compute $\operatorname{Tor}_{i}^{R}(M, N)$ as the homology modules of the complex of $S$-modules $0 \rightarrow$ $N^{2} \stackrel{\bar{X} \otimes N}{\longrightarrow} N^{4} \stackrel{\bar{Y} \otimes N}{\longrightarrow} N^{8}$. Then it is easy to see that the image of $\bar{X} \otimes N$ is $(s, t) N^{4}$ and the image of $\bar{Y} \otimes N$ is $(s, t) N^{8}$. As the length of $N^{4}$ is 12 , this forces the sequence to be exact at $N^{4}$, and so $\operatorname{Tor}_{1}^{R}(M, N)=0$. On the other hand, $\operatorname{Tor}_{2}^{R}(M, N)=(s, t) N^{2} \neq 0$.

Proof of Lemma 1. Begin with generic matrices $X, Y$ of dimensions $2 \times 4$ and $4 \times 8$ respectively. Let $K$ be a field, let $R_{1}=K\left[x_{i j}, y_{i j}\right]$, and let $J$ be the ideal of $R_{1}$ generated by the entries of $X Y$ and all $3 \times 3$ minors of $Y$. Let $R_{2}=R_{1} / J$. This is the ring which Bruns calls $K((2,4,8),(2,2))[2, \mathrm{pp}$. 53-55]. Let $f$ be the $2 \times 2$ minor of $X$ determined by columns 3 and 4, and let $\left\{g_{\sigma}\right\}$ be the set of all $2 \times 2$ minors of $Y$ which use the first two rows (so there is one $g_{\sigma}$ for each pair of columns). Let $R=R_{2}\left[\left\{g_{\sigma} / f\right\}\right]$. It is clear from [2] that $0 \rightarrow R^{2} \stackrel{X}{\longrightarrow} R^{4} \stackrel{Y}{\longrightarrow} R^{8}$ is exact. (The elements $g_{\sigma} / f$ are the $u$-elements in Bruns's paper.)

Next, we claim $R$ is a graded ring. Let $\operatorname{deg}\left(x_{i j}\right)=2$ for each $i, j$, and let $\operatorname{deg}\left(y_{i j}\right)=3$ and $\operatorname{deg}\left(g_{\sigma} / f\right)=2$. Then it is clear that $R$ is a graded polynomial ring modulo a homogeneous ideal and so is graded. Let $P$ be the elements of positive degree. Then $R / P=K$ and $P$ is maximal. Finally, all of the relations between the generators have degree at least 4 , and so $\left\{\bar{x}_{i j}, \bar{y}_{i j}\right\}$ is a linearly independent subset of $P / P^{2}$.

Remarks. The salient features of the example are unchanged if we localize at $P$ or localize and complete.

The Betti numbers (ranks of the free modules in the projective resolution) of $M$ are $\langle 842\rangle$. A similar example can be constructed with Betti numbers $\langle 931$ ); in that case, $N$ will be a module of length 4 .

In [3, p. 287] it was conjectured that rigidity was false and a counterexample might exist with Betti numbers $\langle b \quad b \quad 1\rangle$ for $b>2$. We do not rule this out. However, such an example will necessarily be more complex. The length of $N$ must be considerably larger, and $N$ will not be annihilated by $P^{2}$ or even $P^{3}$.

\section{ACKNOWLEDGMENT}

The author thanks David Eisenbud, Mel Hochster, and Craig Huneke for their assistance in the preparation of this article.

\section{REFERENCES}

1. M. Auslander, Modules over unramified regular local rings, Illinois J. Math. 5 (1961), 631645.

2. W. Bruns, Divisors on varieties of complexes, Math. Ann. 264 (1983), 53-71.

3. S. Dutta, M. Hochster, and J. McLaughlin, Modules of finite projective dimension with negative intersection multiplicities, Invent. Math. 79 (1985), 253-291.

4. M. Hochster, Topics in the homological theory of modules over commutative rings, CBMS Regional Conf. Ser. in Math., vol. 24, Amer. Math. Soc., Providence, RI, 1975. 
5. S. Lichtenbaum, On the vanishing of Tor in regular local rings, Illinois J. Math. 10 (1966), 220-226.

6. C. Peskine and L. Szpiro, Dimension projective finie et cohomologie locale, Inst. Hautes Études Sci. Publ. Math. 42 (1973), 47-119.

7. P. Roberts, The vanishing of intersection multiplicities of perfect complexes, Bull. Amer. Math. Soc. 13 (1985), 127-130.

8. _ Intersection theorems, Commutative Algebra, Math. Sci. Res. Inst. Publ., vol. 15, Springer-Verlag, New York, 1989, pp. 417-436.

Department of Mathematics, University of Texas, Austin, TeXas 78712

E-mail address: heitmann@math.utexas.edu 